\title{
Innovative Technologies in Healthcare, Beware of the Pitfalls
}

In its 2014 opinion on 'the future EU Agenda on quality of healthcare with a special emphasis on patient safety', 2 the Expert Panel on Effective Ways of Investing in Health $(\mathrm{EXPH})^{3}$ indicates five dimensions of healthcare quality:

1. effective, and improve health outcomes

2. Safe, and prevent avoidable harm related with care;

3. Appropriate, and comply with current medical knowledge as well as meeting agreed standards;

4. Patient-centred, and involve patients/people as key partners in the process of care;

5. Efficient, and equitable, and lead to the best value for the money spent and to equal access to available care for equal need, utilisation and quality of care for all.

To achieve these objectives, present days focus is very much on digital health technologies. It is assumed that the use of these technologies will improve efficiency and quality of healthcare.

1 This editorial is based on the keynote speech presented at the 7 th conference of the EAHL, held in Toulouse in September 2019. See also on the subject of artificial intelligence in healthcare, Titti Matson, 'Digitalisation and Artificiel Intelligence in European Healthcare', Eur. J. Health L. 26(4) (2019) 285-288.

2 Specifically, the EXPH was asked to consider the core dimensions of quality of healthcare, including patient safety in the EU; to define the dimensions that should be given priority at EU level in order to improve quality of healthcare, as well as the actions that could be taken at EU level to address the selected dimensions; to demonstrate what would be the added value of proposed EU actions; to specify what information is needed to assess quality and safety of healthcare in the EU. These issues were considered by the EXPH in the context of the Directive on Cross Border Health Care (Directive 2011/24/EU). EXPH, Final Report on future EU Agenda on quality of healthcare with a special emphasis on patient safety, 9 October 2014.

3 The Expert Panel on Effective Ways of Investing in Health (EXPH) was set up by the European Commission Decision 2012/C 198/06. 
Automating tasks and analysing big patient data sets deliver faster and better healthcare at a lower cost. Emerging digital technologies help the system move from episodic to collaborative and longitudinal care. ${ }^{5}$ Information and communication technologies (ICT) allow for the development of decision supportsystems that strengthen the diagnostic capacities of individual practitioners. ${ }^{6}$ AI and health apps enable improved and faster diagnostics. Expert systems ${ }^{7}$ may assist in patient treatments by improving treatment plans and monitoring treatment successes. Care robots may perform automated care tasks, thereby facilitating the work of care assistants. With robotic assisted surgery surgeons may do certain procedures in a minimal invasive way, otherwise done by open surgery.

It is important to keep in mind that robots cannot (and should not) replace the human factor. Adequate training of health professionals working with medical (and care) robots is key for its success.

Another advantage of AI in healthcare, is that a sensor ${ }^{8}$ can detect events or changes in its environment and send the information to other electronics such as a computer processor. This way, a network of intelligent sensors is created that is able to collect, process, transfer and analyse valuable information in different environments. A pertinent example is connecting in-home monitoring devices to hospital-based systems.

4 Digital health is the convergence of digital technologies with health, healthcare, living, and society to enhance the efficiency of healthcare delivery and make medicine more personalised and precise. Artificial intelligence is intelligence exhibited by machines. 'The activity of simulating uniquely human activity and skills', Wikipedia, last accessed on 13 November 2019. See also Yorick Wilks, Artificial Intelligence: Modern Magic or Dangerous Future? (London: Icon books Ltd, 2019).

5 Deloitte, 'A journey towards smart health, the impact of digitalisation on patient experience', February 2018, online at: https://www2.deloitte.com/content/dam/Deloitte/lu/Documents/ life-sciences-health-care/lu_journey-smart-health-digitalisation.pdf.

6 The opinions adopted by EGE, 1998-2000, 'Ethical issues of HealthCare in the Information Society', 30 July 1999, p. 95.

7 An expert system is a computer system that emulates the decision-making ability of a human expert. A legal expert system is a domain-specific expert system that uses artificial intelligence to emulate the decision-making abilities of a human expert in a field of law.

8 A sensor is a device, module, machine, or subsystem whose purpose is to detect events or changes in its environment and send the information to other electronics, frequently a computer processor. 
E- and $m$-health ${ }^{9}$ have the advantage of providing more focused, personalised medicine. ${ }^{10}$ An example of a successful application of these technologies is their assistance to combat substance abuse. Internet based drug dependence treatment ${ }^{22}$ is promoted in the framework of the EC's action plan on e-health 2010-2020. ${ }^{11}$ It is also part of the substance abuse program of the World Health Organisation.

\section{3}

\section{Artificial Intelligence: EU Legal Regime}

\subsection{Medical Devices Regulation}

From a legal perspective a wide variety of issues has to be considered in the development and implementation of digital solutions in healthcare. A key question in the development of software solutions is the regulatory classification of the product. This is of importance in relation to the EU Medical Devices Regulation (MDR). ${ }^{12}$ Medical devices can only be marketed in the EU if they carry a CE label. Such a CE label indicates conformity with health, safety, and environmental protection standards for products sold within the European Economic Area. To acquire the CE label, a positive outcome of a conformity assessment procedure is required. If a product that qualifies as a medical device does not have a CE labelling, a competitor could demand that the product be withdrawn from the market.

According to the MDR the intended purpose of the software is decisive for the classification of a device. Broadly stated this means that in case the software is intended to detect or treat diseases, there is a strong argument for classifying it as a medical device. This is for example the case when the software assists in diagnosis, facilitates decision-making on therapeutic measures, or calculates the dosage of medication. But when the software only provides knowledge or only stores data, it does not qualify as a medical device. It is the manufacturer who determines the intended purpose of the software.

$9 \quad$ E-health refers to healthcare practice supported by electronic processes such as an electronic health record, patient administration and lab systems. M-health is the use of mobile devices (such as a mobile phone or tablet) to support the practice of healthcare.

10 The application of e- and $\mathrm{m}$ - health must comply with the usual quality and safety standards.

11 European Commission, Communication from the European Commissioin to the European Parliament, the Council, the European Social Committee and the Committee of the Regions, 'Digital Single Market, E-Health Action plan 2012-220, Innovative Health Care for the 21st Century', Brussels 6-12-2012.

12 Medical Devices Regulation 2017/745; and In Vitro Diagnostic Regulation 2017/46 (entry into force $25^{-5}-2020$ ). 


\section{2}

Data Protection

3.2.1 General

Data protection is of high relevance in the context of AI. AI solutions are generally based on analysing and comparing specific patient data with a large number of mostly anonymised and aggregated data from other patients. When the data are not anonymised, consent for the collection and use of personal data is required in accordance with the European General Data Protection Regulation (GDPR). ${ }^{13}$ When health data are transmitted outside the device, the processing of health data by lifestyle and well-being apps to be legitimate requires explicit consent.

In 2016, the EU Article 29 Working Party indicated that 'defining the category of health data is important to determine in what circumstances the data processed by lifestyle and wellbeing apps and devices are to be considered data about health.14 The reason for this is that the regime for the processing of health data is more restrictive than the regime for the processing of other types of personal data. In most cases explicit consent is required before health data can be processed, unless national law expressly provides that consent can never justify the processing of such data. ${ }^{15}$ But when the processing relates to data which are manifestly made public by the data subject, explicit consent is not required.

Connectivity of medical devices may have its advantages, it can also have negative implications for patient's privacy and safety. A lack of infrastructure and interoperability standards as well as the likelihood of mechanical failures may cause dis-functioning of the system.

While health and care organisations make sure that sensitive data are stored in a secure and encrypted manner, they do not have control over the security

\footnotetext{
13 The GDPR entered into force in May 2018. The European Data Protection Board (EDPB) an independent European body - contributes to the consistent application of data protection rules throughout the European Union, and promotes cooperation between the EU's data protection authorities. The Board advises the European Commission on any issue related to data protection in the EU, including on any proposed amendment of the GDPR and any EU legislative proposal.

14 February 2015.

15 Article 8 of the GDPR.
} 
of the data access points ${ }^{16}$ used to transmit the data. This brings privacy risks. These risks increase the more the number of devices is connected to the network. For these reasons, doctors and hospitals are looking for solutions to streamline Electronic Health Record (EHR) documentation and to address cybersecurity concerns in relation to patients' health data.

\subsection{Cyber-security}

The primary concern for regulatory bodies is the security of health information, stored and conveyed through connected devices. As indicated by Nicole Wetsman, 'these technologies are often vulnerable to cyberattacks, which can siphon off patient data, hijack drug infusion devices, mine cryptocurrency, or shut down an entire hospital until a ransom is paid. ${ }^{17}$ 'When systems are disrupted over the internet, by an adversary or an accident, that can have a profound impact on patient care.' 18

In December 2018, EU negotiators reached a political agreement on the 2017 Cybersecurity Act which reinforces the mandate of the EU Agency for Cybersecurity. ${ }^{19}$ Medical devices are explicitly mentioned in the Act. It is the aim to better support member states in tackling cybersecurity threats and attacks. An EU framework for cybersecurity certification of specific ICT processes, products, and services will be established.

\subsection{Block Chain Technology}

Protecting data is vital in healthcare. The cost of breach could cost a human life. For these reasons, doctors and hospitals are looking for solutions to streamline EHR documentation and address cybersecurity concerns around patient health data. At the moment, Block-Chain Technology seems to be the most popular strategy. Block-Chain disaggregates the data. It is safe because digital transactions are stored in a distributed, shared ledger. This means that hacking one block in the chain is impossible without simultaneous hacking every other block in the chain's chronology. It passes information from A to B in a fully automated, and safe manner. Digital information will be distributed, but not copied.

\footnotetext{
16 An access point is a wireless network device that acts as a portal for devices to connect to a local area network.

17 Nicole Wetsman, 'Health care's huge cybersecurity problem, Cyberattacks aren't just going after your data', The Verge, 4 April 2019, https://www.theverge.com/2019/4/4/18293817/ cybersecurity-hospitals-health-care-scan-simulation.

18 Beau Woods, a cybersecurity advocate and cyber-safety innovation fellow with the Atlantic Council, cited by Nicole Wetsman.

19 European Union Agency for Network and Information and Security, ENISA
} 
Block-chain appeals to doctors and hospitals because it would enable secure access to a patient's entire health history without compromising security.

\section{$5 \quad$ Civil Law and Robotics}

\subsection{General}

In civil law a large number of issues relating to $\mathrm{AI}$ is relevant. This is in particular the case with AI based robotics. The issues include the validity of contracts, liability for errors and injuries, and matters of insurance. A risk unique to the robotic system is the potential for human error when robotic technology is applied for operating a patient. In general, surgical outcomes are ultimately a direct manifestation of the skills and experience of the surgeon, rather than the technology itself or the approach used. For the sake of patient-safety, robotic surgery requires training and credentialing practices of the medical staff, reporting systems for robotic-associated adverse events and enhanced patient education. ${ }^{20}$ Where patients often lack knowledge and skills about the use of innovative technologies, their application in healthcare requires clear, user friendly explanations.

\subsection{Civil Liability}

The 2017 European Parliament Resolution on Civil Law on Robotics ${ }^{21}$ calls for legal solutions for civil liability caused by robots. It recommends to create a specific legal status for robots as 'electronic persons' responsible for making good any damage they may cause. The Parliament also suggests an EU registration system for advanced robots for specific categories. ${ }^{22}$ At the same time the Resolution emphasises that robot technology should focus on complementing human capabilities and not on replacing them. ${ }^{23}$

According to Aida Ponce Del Castillo, a senior researcher at the European Trade Institute, a wider approach should be adopted by the European Commission, 'encompassing algorithms and AI, and take into consideration machines that have the capacity to learn, evolve and eventually become semior, maybe one day, fully autonomous'. According to Del Castillo, the term 'artificial agent' as a spectrum concept would be more fit for purpose. 'An effective

20 Health professionals should be properly trained for working with medical and care robots.

21 Report with recommendations to the Commission on civil rules on robotics, EP Resolution 16 February 2017.

22 General principles, para. 2.

23 General principles concerning the development of robotics and AI for civil use, para. 3. 
regulatory framework is ultimately required in order to ensure that Artificial Agents co-exist harmoniously with humans and that they are specifically designed for, operate according to and are capable of adapting to human values and needs.... Regulation should ensure that we have visibility and traceability over who is responsible for what.... Artificial agents and humans should coexist, but the former should remain useful to humans, while respecting human value and the rules of democratic societies. ${ }^{24}$

\section{Conclusions}

AI in healthcare brings a number of benefits, including automating tasks and analysing big patient data sets to deliver better healthcare faster, and at a lower cost. AI can be used to help people stay healthy, for early prediction, detection, diagnostic and decision-making support.

As technology continues to evolve and the interconnectedness of devices continues to progress, on a global scale, designing robust cybersecurity protocols at both the device and regulatory body levels will be crucial.

Med-tech companies need to follow key principles of data management and consent that give patients control over their own data. For this purpose governments should initiate a code of conduct for safe, ethical and effective data-driven health- and care technology like the one that was developed by the United Kingdom in 2019. ${ }^{25}$

Such a code must include the ethical principles for data initiatives developed by the Nuffield Council on Bioethics: respect for persons and for human rights, participation and accounting for decisions. ${ }^{26}$

Legal clarity on the rules and norms applicable to the deployment of new digital health technologies is essential. Medical devices should be allowed only on the market when probable benefits to patients outweigh the probable risks.

The potential for AI in healthcare is huge. The unstoppable progress of digitisation will ensure that applications based on AI will increasingly be used in day-to-day treatment. From the outset, those actively engaged in this field, should not only consider the technical aspects but also, and

\footnotetext{
24 Aida Ponce Del Castillo, Foresight Brief, 02, September 2017, pp. 6 and 8.

25 United Kingdom's Code of conduct for data-driven health and care technology of 18 July 2019.

26 Nuffield Council on Bioethics, Bioethics briefing note: Artificial Intelligence (AI) in health care and research, 15 May 208, p. 6.
} 
especially, the legal issues. In this way, it will be possible to analyse, evaluate and cushion the legal risks. Who knows - perhaps an application using legal tech could help with this, since it is well-known that AI is also gaining ground in the legal world.... ${ }^{27}$

Here lays a real challenge for health lawyers.

Henriette Roscam Abbing

Professor Emerita of Health Law, University of Utrecht, Utrecht, The Netherlands; Editor, European Journal of Health Law

27 Roland Wiring, Hamburg, September 2018. 